\title{
EDITORIAL
}

\section{Imaging in the Diagnosis of Lung Disease}

\author{
More Sophisticated Methods Require Greater Interdisciplinary Collaboration
}

Tobias Welte

Editorial to accompany the article

"Radiological diagnosis in lung disease-factoring treatment options into the choice of diagnostic modality" by Mark 0. Wielpütz et al. in this issue of Deutsches Ärzteblatt International
$T$ he continuing development of established techniques such as computed tomography (CT) and magnetic resonance imaging (MRI) has markedly improved the diagnosis of lung disease. With the optimization of the technology, however, new distinctions and specialized variations have come into existence within the imaging techniques that make completely new demands on the collaboration between radiologists and pneumologists, and on the interdisciplinary knowledge of both their disciplines that this collaboration requires.

In this issue, Wielpütz et al. (1) describe which diagnostic techniques should be used for which lung diseases today.

\section{Applications of different techniques}

The advantages of the classical chest radiograph are that it entails a low radiation dose and it is low cost. This is why the chest X-ray is still the standard technique for initial investigations, especially in cases of infectious, malignant, or obstructive airways disease (2).

Although the radiation dose associated with CT studies has been much reduced in recent years, it still accumulates over the course of serial investigations, and thus contributes to cancer risk, at least in at-risk patients (3).

At present there are no convincing results that would allow a positive estimate of the risk-benefit ratio of CT used as a screening method. The advantage in early recognition of bronchial carcinoma - at least in highrisk patients with a long history of smoking - is offset by the disadvantage of frequent false-positive findings, which result in further diagnostic procedures (with their attendant costs) and, for the patient, also in considerable mental stress. The authors of a recent Cochrane Review (4) see insufficient evidence to support a program of bronchial cancer screening using CT and/or sputum cytology. Other large-scale studies on this topic will be completed in the near future. Whether these will change the balance of the risk-benefit assessment, however, remains to be seen.

\section{Computed tomography}

Computed tomography has been familiar for years as a standard procedure in the diagnosis of many lung diseases. Exactly which CT technique is used, however, varies greatly between diseases that can be very different:

- Acute pulmonary embolism

- Chronic pulmonary hypertension

- Interstitial lung disease

- Lung infection

- Bronchial carcinoma (staging)

- Emphysema (quantification).

Physicians often have little knowledge of the great variety of imaging methods available. It might seem obvious that, to be able to perform a targeted diagnostic study, radiologists need a detailed description of the clinical symptoms and of what is wanted from the CT examination-but, sadly, in routine clinical practice they do not always get it.

Most continuing education courses in internal medicine no longer include time for radiology training, and the information provided on the radiation protection course about imaging techniques specific to lung disease is inadequate. My own experience is that studies often have to be repeated because the CT diagnostic technique used the first time around was not the right one for the diagnostic question.

\section{Magnetic resonance imaging}

Magnetic resonance imaging is a promising alternative, especially for serial studies, because of the absence of ionizing radiation. MRI has a role in the diagnostic investigation of vascular diseases such as pulmonary hypertension, or complex diseases such as cystic fibrosis.

The detailed representation of interstitial structures, as needed for differential diagnosis of connective tissue diseases of the lung, remains the strength of CT. It is possible that technical improvements will extend the role of MRI into this area; the only question is, what will it cost?

\section{Future perspectives}

Wielpütz and co-authors present the differential indications for various imaging techniques in the diagnosis of pulmonary and bronchial diseases. However, imaging techniques will in future find applications in areas beyond clinical diagnosis.

For example, imaging techniques could play an important part in future clinical studies, because they help to visualize drug-related changes in the broncho- 
pulmonary system faster and more accurately than was previously possible. The endpoints used in clinical studies today, such as lung function parameters, only show slow changes, thus necessitating long-term studies to show drug effects. Showing the changes through imaging techniques might achieve the same purpose more quickly and more quantifiably.

Shortening the duration of clinical studies would help to win time for patients. Effective drugs would be available sooner, and considerable costs would be saved-so long as surrogate parameters can be found. To support this development, interdisciplinary translational research facilities have been established in Heidelberg, Hannover, and Munich within the framework of the German Center for Lung Research (Deutsches Zentrum für Lungenforschung), funded by the Federal Ministry of Education and Research (Bundesministerium für Bildung und Forschung).

In the diagnosis of lung disease, the future, more even than today, belongs to imaging - both clinically and in research. To make the best use of this, closer collaboration between clinical physicians and radiologists will be important.

Conflict of interest statement

The author declares that no conflict of interest exists.

Translated from the original German by Kersti Wagstaff, MA.

\section{REFERENCES}

1. Wielpütz MO, Heußel CP, Herth FJF, Kauczor HU: Radiological diagnosis in lung disease-factoring treatment options into the choice of diagnostic modality. Dtsch Arztebl Int 2014; 111(11): 181-7.

2. Dalhoff K, Ewig S; on behalf of the Guideline Development Group: Clinical Practice Guideline: Adult patients with nosocomial pneumonia-epidemiology, diagnosis and treatment. Dtsch Arztebl Int 2013; 110(38): 634-40.

3. Sodickson A, Baeyens PF, Andriole KP, et al.: Recurrent CT, cumulative radiation exposure, and associated radiation-induced cancer risks from CT of adults. Radiology 2009; 251: 175-84.

4. Manser R, Lethaby A, Irving LB, et al.: Screening for lung cancer. Cochrane Database Syst Rev 2013; 6: CD001991. doi:10.1002/14651858.CD001991.pub3.

Corresponding author:

Prof. Dr. med. Tobias Welte

Klinik für Pneumologie

Medizinische Hochschule Hannover

Carl-Neuberg-Str. 1

30625 Hannover, Germany

Cite this as:

Welte T: Imaging in the diagnosis of lung disease: more sophisticated methods require greater interdisciplinary collaboration. Dtsch Arztebl Int 2014; 111(11): 179-80.DOl: 10.3238/arztebl.2014.0179 